\title{
Determining the Orientation Parameters of the ICRS/UCAC2 System Using the Kharkov Catalog of Absolute Stellar Proper Motions
}

\author{
V.V. Bobylev ${ }^{1}$, P.N. Fedorov ${ }^{2}$, A.T. Bajkova ${ }^{1}$, and V.S. Akhmetov ${ }^{2}$ \\ ${ }^{1}$ Pulkovo Astronomical Observatory, Russian Academy of Sciences, St-Petersburg \\ ${ }^{2}$ Karazin Kharkov National University, ul. Sumskaya 35, Kharkov, 61022 Ukraine
}

\begin{abstract}
The absolute proper motions of about 275 million stars from the Kharkov XPM catalog have been obtained by comparing their positions in the 2MASS and USNOA2.0 catalogs with an epoch difference of about $45 \mathrm{yr}$ for northern-hemisphere stars and about $17 \mathrm{yr}$ for southern-hemisphere stars. The zero point of the system of absolute proper motions has been determined using 1.45 million galaxies. The equatorial components of the residual rotation vector of the ICRS/UCAC2 coordinate system relative to the system of extragalactic sources have been determined by comparing the XPM and UCAC2 stellar proper motions: $\omega_{x, y, z}=(-0.06,0.17,-0.84) \pm(0.15,0.14,0.14)$ mas $\mathrm{yr}^{-1}$. These parameters have been calculated using about 1 million faintest UCAC2 stars with magnitudes $R_{U C A C 2}>$ $16^{m}$ and $J>14^{m} .7$,for which the color and magnitude equation effects are negligible.
\end{abstract}

\section{INTRODUCTION}

Since 1998, the International Celestial Reference System (ICRS) has been realized by the International Celestial Reference Frame (ICRS), which is represented by the catalog of positions of quasars and other extragalactic radio sources. This catalog was extended to the optical range by the HIPPARCOS and Tycho Catalogue (1997).

Kovalevsky et al. (1997) established that the ICRS/HIPPARCOS coordinate system had no residual rotation relative to an inertial coordinate system with an error of $\pm 0.25 \mathrm{mas} \mathrm{yr}^{-1}$ along three axes.

Subsequently, as new data became available, Bobylev (2004b) found the following rotational parameters of the HIPPARCOS system relative to the extragalactic coordinate system: $\omega_{x}=0.04 \pm 0.15$ mas yr${ }^{-1}, \omega_{y}=0.18 \pm 0.12 \operatorname{mas~yr}^{-1}$, and $\omega_{z}=-0.35 \pm 0.09 \mathrm{mas} \mathrm{yr}^{-1}$ (the rotation components in the equatorial coordinate system). This result is confirmed by a kinematic analysis of HIPPARCOS stars (Bobylev 2004a) and hundreds of thousands of faint Tycho-2 and UCAC2 stars (Bobylev and Khovritchev 2006). On the whole, it is also consistent with the results by Boboltz et al. (2007), who analyzed the positions and proper motions of 46 radio stars and obtained the mutual orientation parameters of the optical realization (HIPPARCOS) and the radio system: $e_{x}=-0.4 \pm 2.6$ mas, $e_{y}=0.1 \pm 2.6$ mas, and $e_{z}=-3.2 \pm 2.9$ mas, as well as the components of the residual rotation vector:

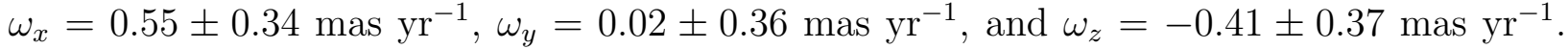
Boboltz et al. (2007) reached the right conclusion that there are no significant rotations 
within the error limits. Note also the estimate of the angular velocity of rotation of the HIPPARCOS system relative the DE403 and DE405 systems of ephemerides obtained by Chernetenko (2008) by analyzing the observations of asteroids: $|\omega|=0.940 .20 \mathrm{mas} \mathrm{yr}^{-1}$, where the components of the vector found are $\omega_{x}=0.12 \pm 0.08 \mathrm{mas} \mathrm{yr}^{-1}, \omega_{y}=0.66 \pm 0.09$

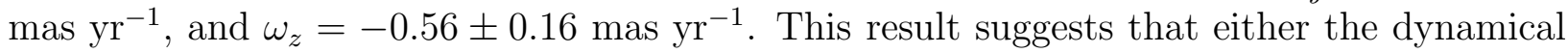
DE403 and DE405 models need to be improved or the HIPPARCOS system needs to be corrected. As we see, one of the components, namely, $\omega_{z}$, differs significantly from zero in several cases. Determining this parameter by an independent method is a very topical problem.

Based on data from the 2MASS (Skrutskie et al. 2006) and USNO-A2.0 (Monet 1998) catalogs, Fedorov et al. (2009) derived the absolute proper motions of about 275 million stars fainter than $12^{m}$ at the Karazin Kharkov National University. For this catalog, we use the abbreviation XPM. The XPM stars cover the entire celestial sphere with the exception of a small region near the Galactic-center direction (Fig. 1b). The stellar proper motions were obtained by comparing the stellar positions in the 2MASS Point Source Catalog and USNO-A2.0 with an epoch difference of about $45 \mathrm{yr}$ for northern-hemisphere stars and about 17 yr for southern-hemisphere stars. The zero point of the system of absolute proper motions (absolutization corrections) was determined using about 1.45 million galaxies from the 2MASS catalog. Thus, the XPM catalog is an independent realization of an inertial coordinate system. The most significant systematic zonal errors in USNO-A2.0 were eliminated before the derivation of proper motions. The mean formal absolutization error is less than

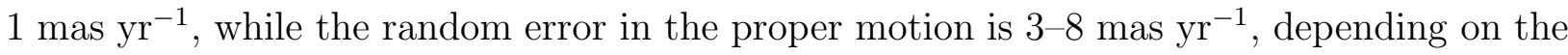
magnitude. The current version of the XPM catalog contains stellar positions in ICRS for the epoch J2000, original absolute stellar proper motions, and B, R, J, H,and K magnitudes.

In this paper, our task is to determine the residual rotation vector of the optical realization of the ICRS/HIPPARCOS system relative to the coordinate system specified by extragalactic sources.

As a realization of the ICRS/HIRRARCOS system, we use the UCAC2 catalog (Zacharias et al. 2004), which extends the system to stars as faint as $R_{U C A C 2} \approx 16^{m} .5$. The XPM catalog of proper motions acts as a realization of an inertial coordinate system. The task is accomplished by comparing the proper motions of common stars in the XPM and UCAC2 catalogs based on which the mutual rotation parameters (the components of vector $\omega$ ) are determined.

\section{THE METHOD}

To determine $\omega\left(\omega_{x}, \omega_{y}, \omega_{z}\right)$, we use the well known equations (Lindgren and Kovalevsky 1995)

$$
\begin{gathered}
\Delta \mu_{\alpha} \cos \delta=\omega_{x} \cos \alpha \sin \delta+\omega_{y} \sin \alpha \sin \delta-\omega_{z} \cos \delta \\
\Delta \mu_{\delta}=-\omega_{x} \sin \alpha+\omega_{y} \cos \alpha
\end{gathered}
$$

where the XPM-UCAC2 stellar proper motion differences are on the left-hand sides. The system of conditional equations (1) and (2) is solved by the least-squares method.

We identified a total of about 36 million common stars in the XPM and UCAC2 catalogs;

$63 \mathrm{XPM}$ fields with unreliable absolutization were excluded-these are located along the Galactic equator, in the zone of avoidance, where there are very few or no galaxies. 
Figure 1 shows an example of the distribution of XPM stars over the celestial sphere. About 1.6 million stars with $B_{U S N O-B}$ magnitudes in the range $17^{m} .00-17^{m} .02$ were used to construct the diagram.

In Fig. 1a, we clearly see a nonuniformity of the distribution due to a strong concentration of stars to the Galactic plane. Obviously, if the system of equations (1) and (2) is solved using individual stars, then the solution will be biased, because the Galactic equator zone, in which an overwhelming majority of stars are located, will have the greatest weight. To get rid of this nonuniformity, we apply Charliers well-known method using equal-area fields. The essence of the method is that despite the difference in the number of stars, a unit weight is assigned to each field when the system of conditional equations (1) and (2) is solved. We divided the sky into 432 fields. Because of various gaps, only about 380 such fields are actually used. The gaps mostly stem from the fact that the UCAC2 catalog contains no data in the northern-sky zone at $\delta>54^{\circ}$.

Comparison of the XPM and UCAC2 stars showed that the mean dispersions of the stellar proper motion differences do not exceed 9 mas $\mathrm{yr}^{-1}$ in both coordinates. In this case, the estimate of $9 / \sqrt{2}=6.4$ mas $\mathrm{yr}^{-1}$ is in good agreement both with the declared accuracy of the UCAC2 proper motions and with the estimates of the accuracy of the XPM proper motions.

To eliminate erroneous identifications, we used the following constraints on the stellar proper motion differences: $\left|\Delta \mu_{\alpha} \cos \delta\right|<50 \mathrm{mas} \mathrm{yr}^{-1}$ and $\left|\Delta \mu_{\delta}\right|<50$ mas yr$^{-1}$.

\section{RESULTS}

\subsection{Analysis of the Differences}

In Fig. 2, the mean XPM.UCAC2 proper motion differences are plotted against the $J$ magnitude; in Fig. 3, the components of the vector $\omega\left(\omega_{x}, \omega_{y}, \omega_{z}\right)$ found from the differences of the same stars are plotted against the $J$ magnitude. About 30 million stars were used to construct Figs. 2 and 3.

We see from Fig. 3 that the components $\omega_{x}$, and $\omega_{y}$ do not differ significantly from zero in the entire range of magnitudes under consideration. In contrast, $\omega_{z}$ depends strongly on the magnitude. Note that $\omega_{z}$ is determined only from Eq. (1) and, hence, its determination is influenced only by differences of the form $\mu_{\alpha} \cos \delta$.

It follows from Fig. 2 that the magnitude dependence of the $\omega_{z}$ differences in the range $12^{m}<J<15^{m}$ can be fitted by a linear trend with a coefficient of the magnitude equation (ME) $-0.6 \pm 0.05$ mas $\mathrm{yr}^{-1}$ per magnitude. This trend can be associated with the presence of ME in the UCAC2 catalog. Indeed, Bobylev and Khovrichev (2006) showed that the proper motions of UCAC2 stars with magnitudes in the range $R_{U C A C 2}=12^{m}-15^{m}$ are distorted by $\mathrm{ME}$ in $\mu_{\alpha} \cos \delta$ with a coefficient of $-0.6 \pm 0.05 \mathrm{mas} \mathrm{yr}^{-1}$ per magnitude. The magnitude dependence of the PUL3SE-UCAC2 differences in $\mu_{\alpha} \cos \delta$ is similar in pattern to that in Fig. 2.

Since Bobylev and Khovrichev (2006) found no noticeable magnitude dependence of the PUL3SE-UCAC2 differences $\mu_{\delta}$, it can be suggested that no ME of this type is present in the XPM catalog. It follows from Fig. 2 that in the range $10^{m}<J<13^{m}$, the ME coefficient in the differences $\mu_{\delta}$ is -0.6 mas $\mathrm{yr}^{-1}$ per magnitude.

Studying ME is the subject of a separate investigation, while to accomplish our task, 
it will suffice to take the faintest UCAC2 stars without any noticeable ME. Stars with $R_{U C A C 2}>16^{m}$ are quite suitable for this purpose. As follows from Fig. 2, bright stars should also be removed to minimize the influence of ME in $\mu_{\delta}$ of the XPM catalog.

In Fig. 4, the components of the vector .found are plotted against the $\mathrm{J}$ magnitude for a sample of stars with $R_{U C A C 2}>16^{m}$. Since there is a constraint on RUCAC2, we observe an increase in color index, for example, $R_{U C A C 2}-J$, with decreasing $J$. As can be seen from the figure, two components, $\omega_{y}$ and $\omega_{z}$, behave stably, while $\omega_{x}$ exhibits a noticeable trend and tends to -1 mas $\mathrm{yr}^{-1}$ for $J<14^{m}$. This behavior of $\omega_{x}$ is related to the presence of a color equation in one of the catalogs being analyzed, because for $J<14^{m}$ the color index, on average, $R_{U C A C 2}-J>2^{m}$, i.e., the stars have a significant reddening. The percentage of such stars is low, but it is better to exclude them from consideration.

The data in Fig. 4 suggest that the influence of both magnitude and color equations is small for the proper motion differences of stars with $J>14^{m}$ and $R_{U C A C 2}>16^{m}$.

To study the influence of the color equation on the color equation is present only in differences of the form quantities being determined, Eqs. (1) and (2) were solved separately. The sample and the approach are the same as those used in constructing Fig. 4, but the magnitude range is wider, $J>13^{m} .0$. The results are reflected in Fig. 5, from which we see that a significant color equations is present only in differences of the form $\Delta \mu_{\alpha} \cos \delta$ and affects mainly the determination of the parameter $\omega_{x}$. It is easy to see that for the dependence of $\omega_{x}$ in the range $R-J=1 \mathrm{~m} .53^{\mathrm{m}}$, the coefficient of the linear trend is $\approx-2.8$ mas $\mathrm{yr}^{-1}$ per magnitude.

Using the differences of 3146504 stars with $J>14^{m} .0$ and $R_{U C A C 2}>16^{m} .0$, we found the following parameters by solving the system of equations (1) and (2):

$$
\begin{gathered}
\omega_{x}=-0.08 \pm 0.14{\operatorname{mas~} \mathrm{yr}^{-1}}^{-1} \\
\omega_{y}=+0.21 \pm 0.14 \mathrm{mas} \mathrm{yr}^{-1}, \\
\omega_{z}=-0.95 \pm 0.13 \text { mas } \mathrm{yr}^{-1} .
\end{gathered}
$$

For this sample, $\bar{R}_{U C A C 2}=16^{m} .2, \bar{J}=14^{m} .8$, and the mean color index $\overline{R-J}=1^{m} .6$.

\subsection{The Kinematics of Sample Stars}

To study the properties of our sample of stars, we found the kinematic parameters of the linear Ogorondikov-Milne model. The method is described in detail in Bobylev and Khovrichev (2006). The conditional equations can be written as

$$
\begin{gathered}
\mu_{l} \cos b=X_{\odot} \sin l-Y_{\odot} \cos l- \\
-M_{32}^{-} \cos l \sin b-M_{13}^{-} \sin l \sin b+M_{21}^{-} \cos b+M_{12}^{+} \cos 2 l \cos b-M_{13}^{+} \sin l \sin b+ \\
+M_{23}^{+} \cos l \sin b-0.5\left(M_{11}^{+}-M_{22}^{+}\right) \sin 2 l \cos b \\
\mu_{b}=X_{\odot} \cos l \sin b+Y_{\odot} \sin l \sin b-Z_{\odot} \cos b \\
+M_{32}^{-} \sin l-M_{13}^{-} \cos l-0.5 M_{12}^{+} \sin 2 l \sin 2 b+M_{13}^{+} \cos l \cos 2 b+ \\
+M_{23}^{+} \sin l \cos 2 b-0.5\left(M_{11}^{+}-M_{22}^{+}\right) \cos ^{2} l \sin 2 b+0.5\left(M_{33}^{+}-M_{22}^{+}\right) \sin 2 b .
\end{gathered}
$$

In this writing (without allowance for the individual distances), all of the sought-for unknowns are expressed in mas $\mathrm{yr}^{-1}$. The Galactic proper motion components averaged in each Charlier field are on the left-hand sides; $l$ and $b$ are the Galactic coordinates. $X \odot, Y \odot$, and $Z \odot$ are the peculiar solar velocity components $M_{12}^{-}, M_{13}^{-}$, and $M_{23}^{-}$are the components 
of the solid-body rotation vector of a small solar neighborhood around the Galactic $Z, Y$ and $X$ axes, respectively. The quantity $M_{12}^{-}\left(\right.$mas $\left.\mathrm{yr}^{-1}\right)$ is related to the Oort constant $B$ $\left(\mathrm{km} \mathrm{s}^{-1} \mathrm{kpc}^{-1}\right)$ via the proportionality coefficient 4.74 .

Each of the quantities $M_{12}^{+}, M_{13}^{+}$, and $M_{23}^{+}$describes the deformation in the corresponding plane. The quantity $M_{12}^{+}\left(\right.$mas $\left.\mathrm{yr}^{-1}\right)$ is related to the Oort constant $A\left(\mathrm{~km} \mathrm{~s}^{-1} \mathrm{kpc}^{-1}\right)$ via the proportionality coefficient 4.74 . The diagonal components of the local deformation tensor $M_{11}^{+}, M_{22}^{+}$, and $M_{33}^{+}$describe the overall contraction or expansion of the entire stellar system. If only the stellar proper motions are used, then one of the diagonal terms of the local deformation tensor is known to remain uncertain. Therefore, we determine differences of the form $\left(M_{11}^{+}-M_{22}^{+}\right)$and $\left(M_{33}^{+}-M_{22}^{+}\right)$.

To estimate the mean distance to the sample stars, we use a statistical method. As the known peculiar solar velocity relative to the local standard of rest, we take the values from Dehnen and Binney (1998): $\left(U_{\odot}, V_{\odot}, W_{\odot}\right)=(10.00,5.25,7.17) \pm(0.36,0.62,0.38) \mathrm{km} \mathrm{s}^{-1}$. We calculate the parallax using two formulas: $\pi_{U}=4.74 \cdot X_{\odot} / U_{\odot}$ and $\pi_{W}=4.74 \cdot Z_{\odot} / W_{\odot}$, where $X_{\odot}$ and $Z_{\odot}$ are the stellar group velocity components found, expressed in mas $\mathrm{yr}^{-1}$. Since the component $Y_{\odot}$ is noticeably distorted by the asymmetric drift velocity (Dehnen and Binney 1998), this projection is not used to determine the group parallaxes. We find the distance $d$ from the relation $d=1 / \pi$. The table gives two estimates obtained from the velocities $U_{\odot}$ and $W_{\odot}$.

The results of solving the system of equations (4) and (5) by the least-squares method are presented in the table. To eliminate the stars with large proper motions that can spoil the statistics, we used a constraint on the magnitude of the tangential stellar velocity, $\sqrt{\left(\mu_{\alpha} \cos \delta\right)^{2}+\left(\mu_{\delta}\right)^{2}}<150$ mas $\mathrm{yr}^{-1}$. The solutions were obtained for the same sample of stars from which solution (3) was found, but with two different sets of stellar proper motions-from the XPM and UCAC2 catalogs.

As follows from the table, all kinematic parameters of the Ogorodnikov-Milne model are determined slightly more accurately from the UCAC2 proper motions. The solutions obtained from UCAC2 are in excellent agreement with their analysis performed by Bobylev and Khovrichev (2006) based on different samples from this catalog. There are slight differences for a number of parameters found using the XPM catalog. These include the solar velocity components $X_{\odot}$ and $Z_{\odot}$ and, as a result, the difference in the estimates of the statistical distance $d_{U}$ and $d_{W}$. There are also differences in the estimates of the Oort constants $A$ and $B$. In compiling our sample of stars for solution (3), we took faint UCAC2 stars, while for the XPM catalog these stars $\left(\bar{B}=16^{m} .6\right)$ are by no means faint (the limiting magnitude is $B \approx 21^{m}$ ). The above differences in these parameters are probably attributable to the presence of small (we got rid of the large ones) magnitude and color equation effects in the XPM catalog, which require their careful study and elimination to obtain reliable data in analyzing the Galactic kinematics.

For the goals of this paper, we are most interested in the values of the parameter $M_{13}^{-}=$

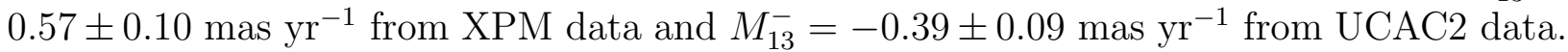

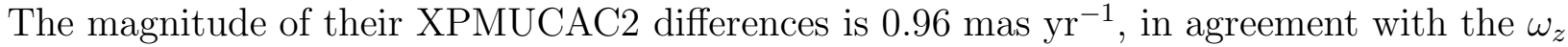
magnitude of solution (3). This is because in the Galactic coordinate system, the direction of the celestial pole is close to the direction of the Galactic $Y$ axis. Therefore, the rotation around the equatorial $Z$ axis manifests itself in the Galactic coordinate system mainly as the rotation around the Galactic $Y$ axis that, in our case, is described by the parameter $M_{13}^{-}$. 


\subsection{The Vector $\omega$.}

Using the proposed constraints, we got rid of the significant manifestations of the magnitude and color equations. However, as we see from Figs. 4 and 5, even for $J>14^{m}$ there are small trends for all of the quantities being determined. Therefore, for the final solution, we used the differences of 1145768 faintest stars with $J>14^{m} .7$ and $R_{U C A C 2}>16^{m} .0$, which, in our opinion, deserve the greatest confidence. In Figs. 4 and 5, two rightmost points correspond to this magnitude range. For this sample, $\bar{J}=14 .^{m} 9$ and the mean color index $\overline{R-J}=1 \cdot^{m} 4$. As a result, we found the parameters

$$
\begin{gathered}
\omega_{x}=-0.06 \pm 0.15 \operatorname{mas~yr}^{-1}, \\
\omega_{y}=+0.17 \pm 0.14 \mathrm{mas} \mathrm{yr}^{-1}, \\
\omega_{z}=-0.84 \pm 0.14 \mathrm{mas} \mathrm{yr}^{-1},
\end{gathered}
$$

which are the main result of this work.

\section{DISCUSSION}

The values of the components $\omega_{x}$ and $\omega_{y}$ we found do not differ significantly from zero. The value of $\omega_{z}=-0.84 \pm 0.14$ mas $\mathrm{yr}^{-1}$ differs significantly from zero. Our results are qualitatively in good agreement with those of several most extensive individual programs used to reference the HIPPARCOS catalog to the system of extragalactic sources (Kovalevsky et al. 1997). For example, the Kiev program (Kislyuk et al. 1997) yielded $\omega_{x}=-0.27 \pm 0.80$ mas $\mathrm{yr}^{-1}, \omega_{y}=+0.15 \pm 0.60$ mas yr${ }^{-1}$, and $\omega_{z}=-1.07 \pm 0.80$ mas $\mathrm{yr}^{-1}$. Note also the results of the Pulkovo program (Bobylev et al. 2004): $\omega_{x}=-0.98 \pm 0.47$ mas yr$^{-1}, \omega_{y}=-0.03 \pm 0.38$ mas $\mathrm{yr}^{-1}$, and $\omega_{z}=-1.66 \pm 0.42$ mas $\mathrm{yr}^{-1}$. Our parameters are also in good agreement with the present-day results of the analysis of a long-term series of asteroid observations (Chernetenko 2008) noted in the Introduction.

The manifestations of the magnitude and color equations found in the bright part of the current XPM catalog are attributable to the peculiarities of deriving its proper motions, because the stellar images in the 2MASS and USNO-A2.0 catalogs were obtained in the near-infrared and optical ranges, respectively.

Using a sufficiently large number of comparison stars in solving (4) allowed the soughtfor parameters to be determined with a high accuracy. Our constraints give hope that the results obtained are not distorted by the magnitude and color equation effects.

\subsection{CONCLUSIONS}

Comparison of the intermediate version of the XPM catalog and UCAC2 showed that the mean dispersions of the stellar proper motion differences are $\approx 9$ mas $\mathrm{yr}^{-1}$ in both coordinates. This gives an estimate for the mean random error of the stellar proper motions from

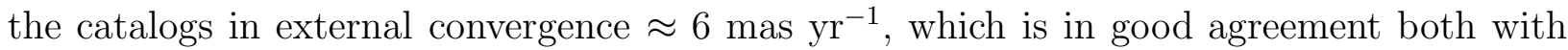
the declared accuracy of UCAC2 and with the estimates of their accuracy in XPM.

We established that the XPM-UCAC2 proper motion differences, both $\Delta \mu_{\alpha} \cos \delta$ and $\Delta \mu_{\delta}$, have significant nonlinear magnitude dependences.

We believe that the magnitude equation in $\Delta \mu_{\alpha} \cos \delta$ and $\Delta \mu_{\delta}$ is related to its presence in the UCAC2 and XPM catalogs, respectively. In the range $10^{m}<J<13^{m}$, the coefficient of the linear trend for the magnitude equation in $\Delta \mu_{\delta}$ is $\approx-0.6$ mas yr$^{-1}$ per magnitude. 
We showed that a significant dependence on color, the coefficient of the linear trend of which in the range $R_{U C A C 2} J=1^{m} .5-3^{m}$ for $\omega_{x}$ is $\approx-2.8$ mas $\mathrm{yr}^{-1}$ per magnitude, is present in $\mu_{\alpha} \cos \delta$. To minimize these effects, we proposed a number of constraints on the magnitude and color of stars. We showed that acceptable parameters $\omega_{x}, \omega_{y}, \omega_{z}$ could be obtained for $J>14^{m} .0$ and $R_{U C A C 2}>16^{m}$ (solution (3)). Our kinematic control based on the linear Ogorodnikov-Milne model indicated that the sample of XPM stars used has no significant kinematic deviations.

The most reliable components of the residual rotation vector of the ICRS/UCAC2 coordinate system relative to the system of extragalactic sources, $\left(\omega_{x}, \omega_{y}, \omega_{z}\right)=$ $(-0.06,0.17,-0.84) \pm(0.15,0.14,0.14)$ mas $\mathrm{yr}^{-1}$, were calculated using 1145768 faintest UCAC2 stars with $R_{U C A C 2}>16^{m}$ and $J>14^{m} .7$.

The components $\omega_{x}, \omega_{y}$, and $\omega_{z}$ found can be used to derive the most probable parameters

of referencing the optical realization of the ICRS/HIPPARCOS system to the system of extragalactic sources.

Of great interest is a further kinematic analysis of the absolute proper motions for faint XPM stars. However, the magnitude and, particularly, color equation effects in this catalog should be carefully studied and removed.

\section{ACKNOWLEDGMENTS}

We are grateful to the referees for useful remarks that contributed to an improvement of this paper. The Russian authors are grateful to G.T. Bajkova for help in working with the data. This study was supported by the Russian Foundation for Basic Research (project no. 09-02-90443-Ukr_f) from the Russian side and by the Contest for joint basic research projects "DFFD-RFFD-2009" (project no. F28.2/042) from the Ukrainian side.

\section{REFERENCES}

D.A. Boboltz, A.L. Fey, W.K. Puatua, et al., Astron. J. 133, 906 (2007).

V.V. Bobylev, Pisma Astron. Zh. 30, 289 (2004a) [Astron. Lett. 30, 251 (2004)].

V.V. Bobylev, Pisma Astron. Zh. 30, 930 (2004b) [Astron. Lett. 30, 785 (2004)].

V.V. Bobylev and M.Yu. Khovrichev, Pisma Astron. Zh. 32, 676 (2006) [Astron. Lett. 32, 608 (2006)].

V.V. Bobylev, N.M. Bronnikova, and N.A. Shakht, Pisma Astron. Zh. 30, 519 (2004) [Astron. Lett. 30, 469 (2004)].

Yu. A. Chernetenko, Pisma Astron. Zh. 34, 296 (2008) [Astron. Lett. 34, 266 (2008)].

W. Dehnen and J.J. Binney, Mon. Not. R. Astron. Soc. 298, 387 (1998).

P.N. Fedorov, A.A. Myznikov, and V.S. Akhmetov, Mon. Not. R. Astron. Soc. 393, 133 (2009).

The HIPPARCOS and Tycho Catalogues, ESA SP-1200 (1997).

V.S. Kislyuk, S.P. Rybka, A.I. Yatsenko, et al., Astron. Astrophys. 321, 660 (1997).

J. Kovalevsky, L. Lindegren, M.A.C. Perryman, et al., Astron. Astrophys. 323, 620 (1997).

L. Lindegren and J. Kovalevsky, Astron. Astrophys. 304, 189 (1995).

D.G. Monet, Bull. Am. Astron. Soc. 30, 1427 (1998).

M.F. Skrutskie, R.M. Cutri, R. Stiening, et al., Astron. J. 131, 1163 (2006).

N. Zacharias, S.E. Urban, M.I. Zacharias, et al., Astron. J. 127, 3043 (2004). 
Table 1: Kinematic parameters of the OgorodnikovMilne model

\begin{tabular}{|c|r|r|}
\hline Parameters & XPM & UCAC2 \\
\hline$X_{\odot}, \mathrm{mas} / \mathrm{yr}$ & $1.53 \pm 0.11$ & $2.55 \pm 0.09$ \\
$Y_{\odot}, \mathrm{mas} / \mathrm{yr}$ & $6.16 \pm 0.10$ & $7.59 \pm 0.09$ \\
$Z_{\odot}, \mathrm{mas} / \mathrm{yr}$ & $1.14 \pm 0.11$ & $2.28 \pm 0.09$ \\
$M_{21}^{+}, \mathrm{mas} / \mathrm{yr}$ & $1.89 \pm 0.14$ & $2.72 \pm 0.12$ \\
$M_{32}^{-}, \mathrm{mas} / \mathrm{yr}$ & $-0.28 \pm 0.11$ & $-0.10 \pm 0.09$ \\
$M_{13}^{-}, \mathrm{mas} / \mathrm{yr}$ & $0.57 \pm 0.10$ & $-0.39 \pm 0.09$ \\
$M_{21}^{-}, \mathrm{mas} / \mathrm{yr}$ & $-2.12 \pm 0.11$ & $-2.39 \pm 0.09$ \\
$M_{11-22}^{+}, \mathrm{mas} / \mathrm{yr}$ & $-0.19 \pm 0.27$ & $-0.21 \pm 0.23$ \\
$M_{13}^{+}, \mathrm{mas} / \mathrm{yr}$ & $-0.16 \pm 0.13$ & $0.10 \pm 0.11$ \\
$M_{23}^{+}, \mathrm{mas} / \mathrm{yr}$ & $0.24 \pm 0.13$ & $-0.06 \pm 0.11$ \\
$M_{33-22}^{+}, \mathrm{mas} / \mathrm{yr}$ & $-0.07 \pm 0.28$ & $0.12 \pm 0.24$ \\
\hline$d_{U}, \mathrm{kpc}$ & $1.4 \pm 0.4$ & $0.8 \pm 0.2$ \\
$d_{W}, \mathrm{kpc}$ & $1.3 \pm 0.4$ & $0.7 \pm 0.2$ \\
$A, \mathrm{~km} / \mathrm{s} / \mathrm{kpc}$ & $8.9 \pm 0.6$ & $12.9 \pm 0.5$ \\
$B, \mathrm{~km} / \mathrm{s} / \mathrm{kpc}$ & $-10.1 \pm 0.5$ & $-11.3 \pm 0.4$ \\
\hline \multicolumn{3}{|c}{}
\end{tabular}




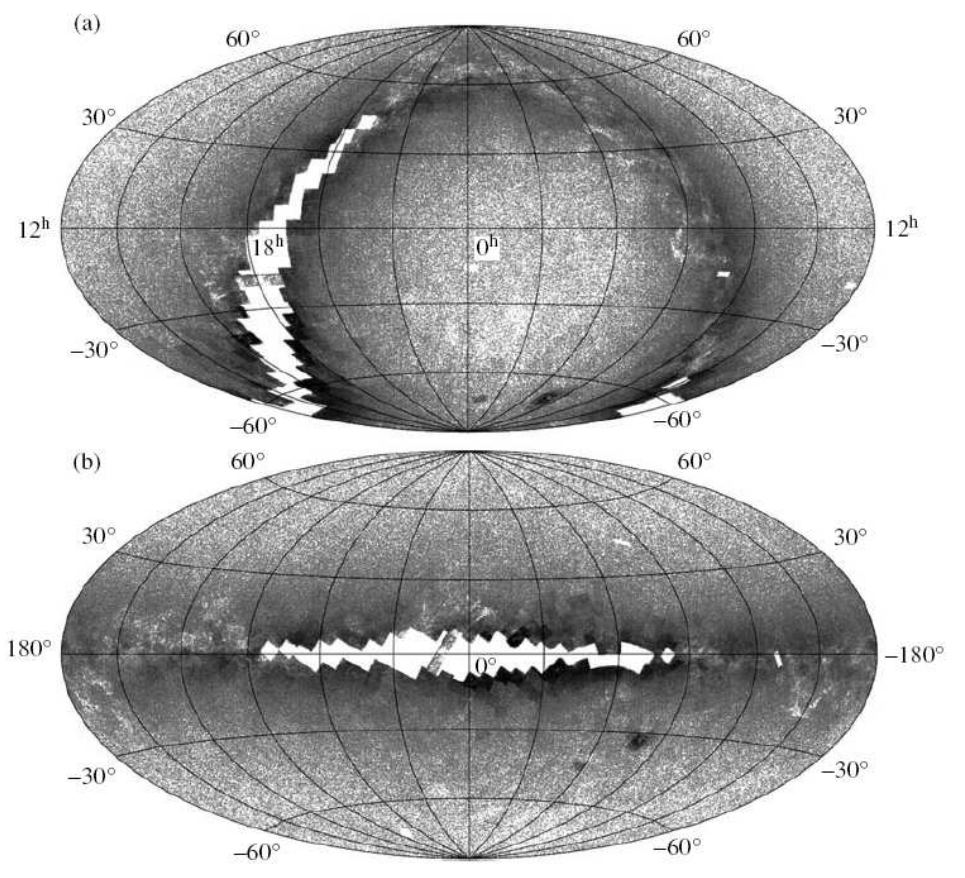

Fig. 1. Distributions of the sample of XPM $B=17^{m}$ stars over the celestial sphere in the equatorial (a) and Galactic (b) coordinate systems. The empty regions mark 63 fields with unreliable absolutization.

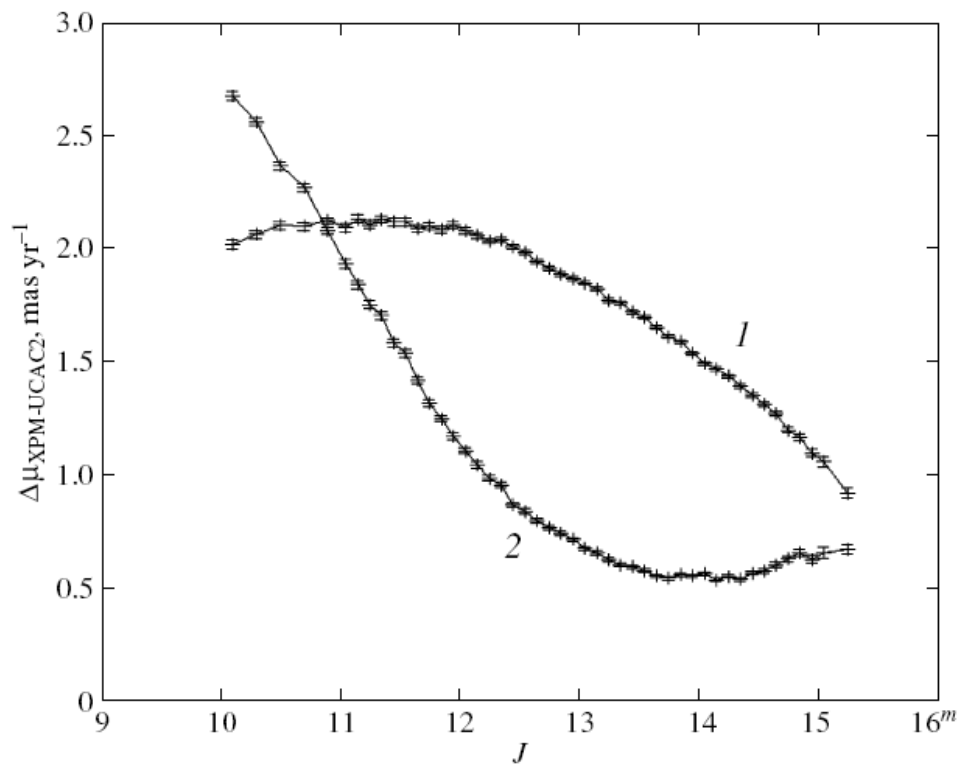

Fig. 2. Mean XPM-UCAC2 stellar proper motion differences $\mu_{\alpha} \cos \delta(1)$ and $\mu_{\delta}(2)$ versus $J$ magnitude. 


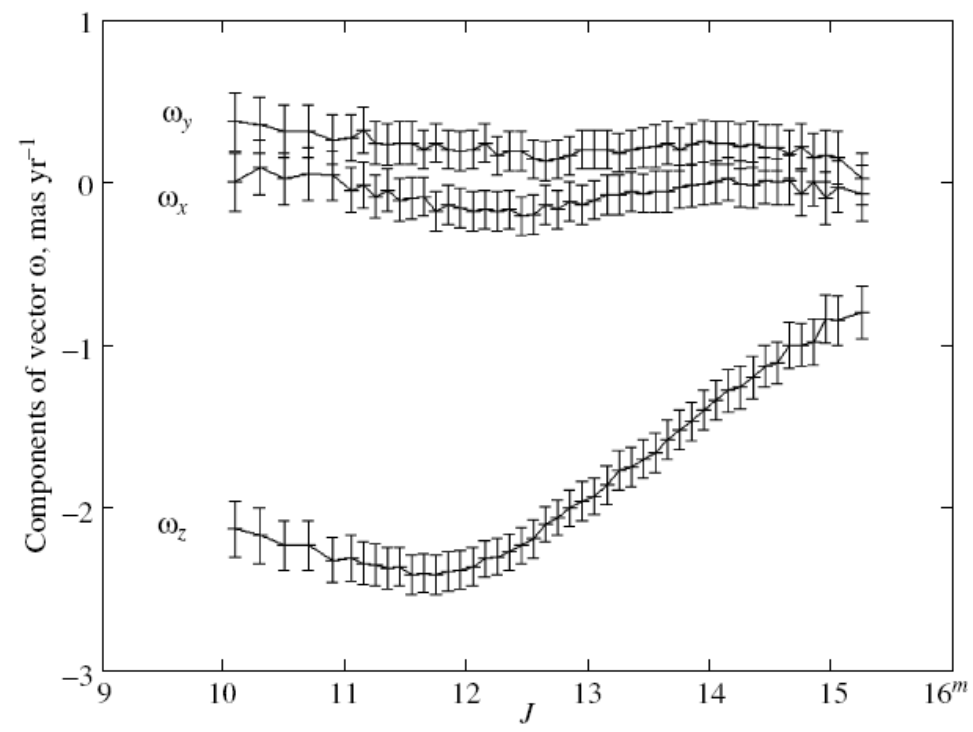

Fig. 3. Components of the residual rotation vector of the ICRS/UCAC2 coordinate system relative to the system of extragalactic objects found by comparing the XPM and UCAC2 stars versus $J$ magnitude.

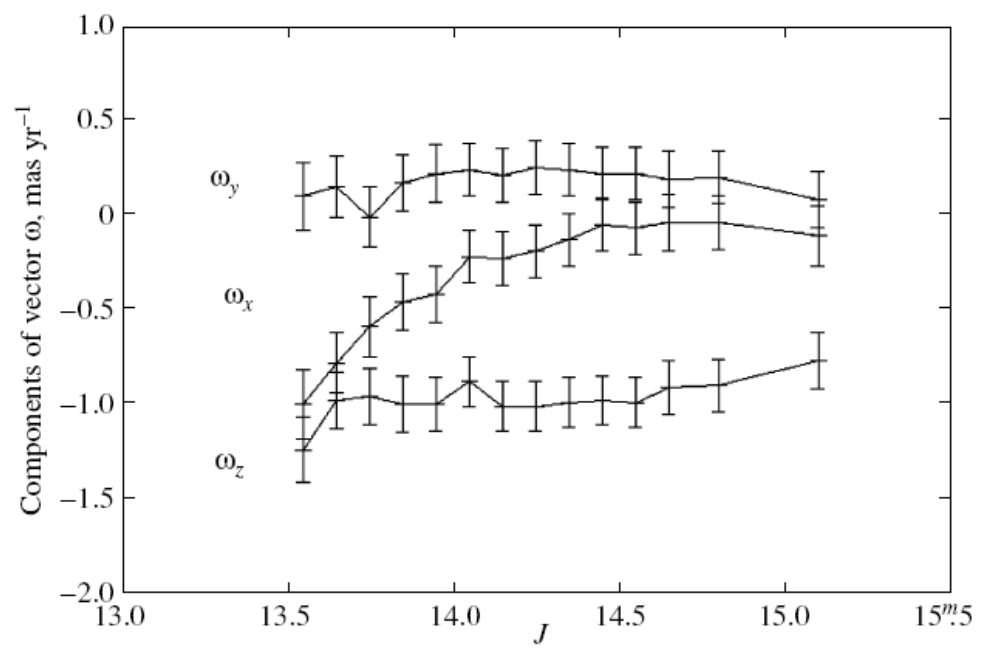

Fig. 4. Components of the residual rotation vector of the ICRS/UCAC2 coordinate system relative to the system of extragalactic objects found by comparing the XPM and UCAC2 stars versus $J$ magnitude for a sample of stars with $R_{U C A C 2}>16^{m}$. 


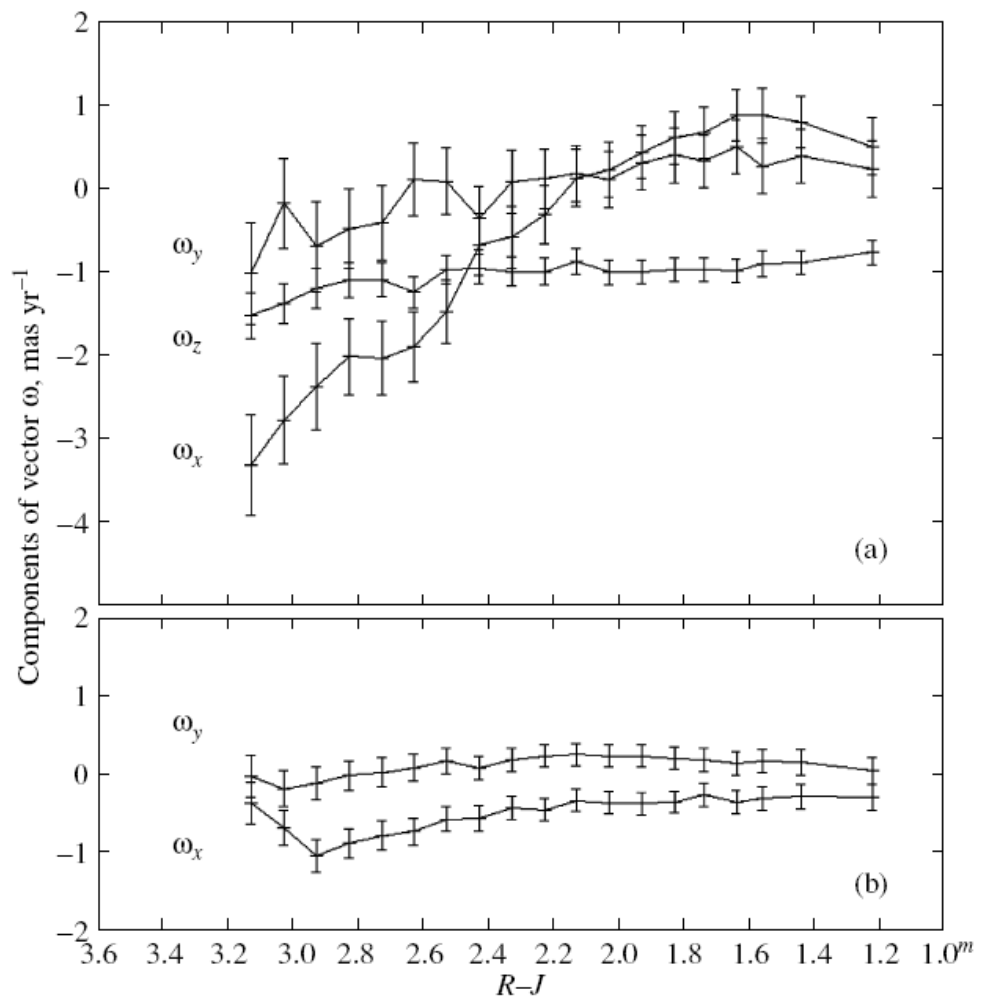

Fig. 5. Components of the vector $\omega$ versus $R_{U C A C 2}-J$ color index found by separately solving Eqs. (1) and (2): (a) only from Eq. (1) and (b) only from Eq. (2). 